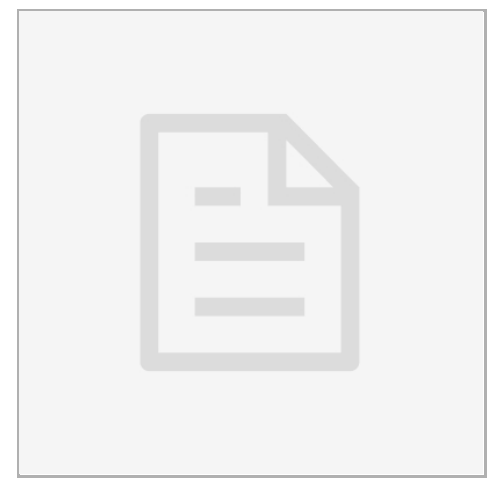

AUG 27, 2018

\title{
(3) Experimental test of the intrinsic dimensionality of Hounsfield unit measurements
}

Zachary Levine $^{1}, \quad$ Adele Peskin ${ }^{1}$, Andrew Holmgren $^{1}$, Edward Garboczi ${ }^{1}$

${ }^{1}$ National Institute of Standards and Technology

Adele Peskin

\section{open ठaccess}

\section{DOI:}

dx.doi.org/10.17504/protocol s.io.sw3efgn

Protocol Citation: Zachary Levine, Adele Peskin, Andrew Holmgren, Edward Garboczi 2018. Experimental test of the intrinsic dimensionality of Hounsfield unit measurements. protocols. io https://dx.doi.org/10.17504/p rotocols.io.sw 3efgn

License: This is an open access protocol distributed under the terms of the Creative Commons Attribution License, which permits unrestricted use, distribution, and reproduction in any medium, provided the original author and source are credited

Protocol status: Working We use this protocol and it's working

Created: Aug 24, 2018

Last Modified: Aug 27, 2018

PROTOCOL integer ID: 15035 


\section{ABSTRACT}

Purpose: The immediate purpose is to determine the maximum number of tube voltages which are useful for acquiring information in medical computed tomography (CT) along with specific linear combinations of quantities of chemical elements which are observable with the current generation of medical CT machines. The larger purpose is to encourage the development of $\mathrm{CT}$ reconstruction algorithms based on the material basis described herein and, ultimately, to tie the measurand of $\mathrm{CT}$ to the International System of Units (SI).

Methods and Materials: Experimentally, we scanned samples at four tube voltages, namely $80 \mathrm{kV}, 100 \mathrm{kV}, 120 \mathrm{kV}$, and $140 \mathrm{kV}$ on a medical CT. The samples included 30 small plastic bottles of powders containing various compounds spanning the atomic numbers from 1 to 20, along with a similar bottle of water and one of air. Using the known chemical formulas and measured masses, we formed a matrix giving the number of Hounsfield units per (mole per cubic meter) at each tube voltage for each of 13 chemical elements. Theoretically, we took the XCOM cross sections, combined them with the tungsten anode spectral model using interpolating cubic splines (TASMICS), with a one-parameter filter, and a simple detector model, and created a similar matrix for the first 20 chemical elements. In the training phase, we found a best-fit parameter. We define the molar Hounsfield unit (HU) potency as the difference in $\mathrm{HU}$ values that an added mole per cubic meter in a given voxel would add to the measured $\mathrm{HU}$ value. We built a matrix of molar potencies for each chemical element and tube voltage and performed a singular value decomposition (SVD) on these. In a validation phase, we compared the model predictions to Hounsfield unit measurements on three CT calibration phantoms taken from the literature.

Results: We found that we could predict the measured HU potency for the chemical elements within our model. The singular values and singular vectors of the model and powder measurements are in substantial agreement. Application of the Bayesian Information Criterion (BIC) shows that exactly two singular values and singular vectors describes the results over four tube voltages. We were able to give a good account of the $\mathrm{HU}$ values measured for the calibration phantoms at several tube voltages for several vendors within our model without introducing additional parameters.

Conclusions: Measurements at two tube voltages are necessary and sufficient to extract the available material dependent information in medical CT. The linear combination of elements that can be observed using a medical CT have been characterized, providing a material basis for use in dual-energy reconstruction. This approach provides groundwork for improved reconstruction and for the link of Hounsfield units to the SI.

\section{ATTACHMENTS}

protocol.docx 
